Research Article

\title{
Salvia spinosa L. Protects against Diabetes-Induced Nephropathy by Attenuation of Mitochondrial Oxidative Damage in Mice
}

\author{
Milad Jeshan (D, , ${ }^{1,2}$ Fatemeh Yousefbeyk ${ }^{D},{ }^{3}$ Hiva Rahmati ${ }^{(D)},{ }^{1,2}$ Amir Hosein Shoormeij, \\ Mitra Rezazadeh, ${ }^{4}$ and Ehsan Zamani $\left(^{2}\right.$ \\ ${ }^{1}$ Student Research Committee, School of Pharmacy, Guilan University of Medical Sciences, Rasht, Iran \\ ${ }^{2}$ Department of Pharmacology and Toxicology, School of Pharmacy, Guilan University of Medical Sciences, Rasht, Iran \\ ${ }^{3}$ Department of Pharmacognosy, School of Pharmacy, Guilan University of Medical Sciences, Rasht, Iran \\ ${ }^{4}$ Laboratory of Pathology, Imam Khomeini Hospital, Jiroft University of Medical Sciences, Jiroft, Iran
}

Correspondence should be addressed to Ehsan Zamani; zamani.ehsan@gums.ac.ir

Received 15 August 2021; Revised 29 November 2021; Accepted 13 December 2021; Published 26 December 2021

Academic Editor: Mohd Esa Norhaizan

Copyright (C) 2021 Milad Jeshan et al. This is an open access article distributed under the Creative Commons Attribution License, which permits unrestricted use, distribution, and reproduction in any medium, provided the original work is properly cited.

Mitochondrial oxidative damage is a crucial factor in the pathogenesis of diabetic nephropathy (DN), which is among the most prevalent problems of diabetes, and there hasn't been an effective treatment for DN yet. This study planned to investigate the effects of Salvia spinosa L. on mitochondrial function along with its protection against streptozotocin-induced nephropathy in diabetic mice. After the injection of streptozotocin (STZ) and verification of the establishment of diabetes, mice $(n=30)$ were randomly divided into the following groups: control group, diabetic-control, S. spinosa-treated diabetic (50, 100, and 200 mg/kg), and metformin-treated diabetic group $(500 \mathrm{mg} / \mathrm{kg})$. After four weeks of treatment, the mice were weighed. Blood and kidney tissues were examined for biochemical and histological evaluation. Hematoxylin and eosin staining was used for evaluating renal pathologic damage. Oxidative damage in the kidney was assessed by the evaluation of lipid peroxidation and glutathione oxidation. Furthermore, differential centrifugation was used to obtain the isolated mitochondria, and mitochondrial toxicity endpoints (mitochondrial function and mitochondrial oxidative markers) were determined in them. S. spinosa remarkably reduced the blood urea and creatinine concentrations, and also normalized kidney weight/body weight coefficient in the diabetic mice. S. spinosa ameliorated the incidence of glomerular and tubular pathological changes in histological analyses. Moreover, the oxidative and mitochondrial damages were notably attenuated in renal tissues of S. spinosa-treated mice. These results indicate that the methanolic extract of $S$. spinosa modulates the nephropathy in the diabetic mice by the amelioration of oxidatively induced mitochondrial damage and provides a reliable scientific base, suggesting S. spinosa as a promising alternative remedy against DN.

\section{Introduction}

Diabetes mellitus (DM) leads to profound pathological complication including diabetic nephropathy ( $\mathrm{DN}$, a microvascular complication), in approximately $25-35 \%$ of patients with both main types of DM $[1,2]$. The progression of DN has various clinical stages, from mesangial hypertrophy and glomerular hyperfiltration with microalbuminuria to macroalbuminuria and nephrotic proteinuria, followed by chronic renal disease, which finally lead to end-stage kidney disease [3].
Regarding the complex pathogenesis of diabetes, the persistent hyperglycemia leads to an excessive reactive oxygen species (ROS) generation, oxidative stress, and mitochondrial dysfunction $[4,5]$. Eventually, oxidative damage occurs in the lipids, proteins, and nucleic acids $[1,6,7]$. Moreover, the ROS play a main role in the pathogenesis of many diseases, and antioxidants are responsible for keeping the normal balance [8].

In clinical practice, controlling excessive oxidative stress, hyperglycemia, dyslipidemia, and hypertension are the important strategies to improve albuminuria and ultimately 
treat DN $[9,10]$. Nowadays, researchers' attention is drawn to natural antioxidants on account of mediocre outcome of classic medications $[7,8]$.

Medicinal herbs have gained significant importance in the last few decades, and available literature shows that huge numbers have demonstrated considerable antioxidant effects on diseases including DN $[2,3,5,10]$.

Salvia spinosa L. is a plant belonging to the Salvia genus, in the Lamiaceae family, which is native to Iran. According to the previous reports, Salvia species are rich in bioactive compounds such as phenolic compounds, terpenoids, isoprenoids, triterpenoids, and sesterterpenoids [11]. These phytochemicals make this genus an appropriate candidate for providing a natural antioxidant $[11,12]$, antibacterial, anti-inflammatory, anticancer, antimalarial, antidiabetic $[11,13]$, and anti-Alzheimer's disease agent [8, 11-14]. For example, a study showed that the aqueous extract of Salvia miltiorrhiza administered to type II diabetic rats significantly decreased lipid peroxidation injury [3].

As regards the mentioned properties of Salvia species, this traditional herb may yield potential candidates for the development of novel therapies against DN. However, till now, the protective effects of $S$. spinosa L. have not been investigated on diabetic nephropathy. Therefore, the aim of this study was to investigate the efficacy of the methanolic extract of S. spinosa L. as nephroprotective in diabetic male mice.

\section{Materials and Methods}

2.1. Preparation of Methanol Extract of Salvia spinosa. Salvia spinosa L. aerial parts were collected from Rostamabad, Guilan, north of Iran (June 2018). The plant specimen was recorded at the herbarium of the School of Pharmacy, Guilan University of Medical Sciences, Rasht, Iran (112 HGUM).

S. spinosa $(500 \mathrm{~g})$ was dried in the shade and powdered. Eventually, we extracted the powder with methanol by the percolation technique. Then, the solvent was evaporated in a rotary evaporator to obtain the extract. Finally, the extracts were refrigerated until other assays.

2.2. Animals. Male albino mice $(n=30)$, weighing $25-30 \mathrm{~g}$, were purchased from the Guilan University of Medical Sciences' animal breeding and care center, Rasht, Iran. The animals were kept under standard conditions with a 12-hour light: 12-hour dark cycle, $45-55 \%$ humidity, at room temperature (RT), and with free access to standard water and food during the study.

All protocols were confirmed by the Ethics Committee of the Guilan University of Medical Sciences (Ethical No.: IR.GUMS.REC.1398.358).

2.3. Diabetes Induction and Experimental Procedure. After overnight fasting, mice were subjected to a single i.p. injection of fresh streptozotocin (STZ) $(45 \mathrm{mg} / \mathrm{kg})$ prepared in sodium citrate buffer ( $\mathrm{pH}$ 4.5). After one week of STZ injection, mice with plasma glucose higher than $250 \mathrm{mg} / \mathrm{dl}$ were considered diabetic mice. Randomly, mice were allocated into six groups $(n=5)$ as follows:

Group I: control (nondiabetic mice treated with normal saline, i.p.)

Group II: diabetic mice (treated with normal saline, i.p.)

Group III-V: diabetic mice treated with S. spinosa doses (i.p., 50, 100, and $200 \mathrm{mg} / \mathrm{kg}$.)

Group VI: diabetic mice treated by metformin (i.p., $500 \mathrm{mg} / \mathrm{kg}$ )

Mice were euthanized by the injection of ketamine $(80 \mathrm{mg} / \mathrm{kg})$ and xylazine $(5 \mathrm{mg} / \mathrm{kg})$ after experiments according to the standard protocols [15].

2.4. Assessment of Fasting Blood Glucose (FBG). At the end of the experimentation, FBG was evaluated by tail vein blood sampling with a glucometer (EasyGluco, Infopia Co., Korea) [16].

2.5. Evaluation of Kidney/Body Weight Coefficient. Body weight of mice was evaluated after overnight fasting. Following euthanization, the kidneys were instantly and precisely weighed. The kidney/body weight coefficient was determined as follows: kidney/body weight coefficient $(\mathrm{g} / \mathrm{g})$ equals kidney weight $(\mathrm{g})$ divided by body weight $(\mathrm{g})$ [2].

2.6. Biochemical Analyses. Blood samples were gained from retro-orbital venous plexus and placed on cold EDTAcontaining tubes. For the separation of sera, the samples were centrifuged at $2300 \times \mathrm{g}$ and stored at $-80^{\circ} \mathrm{C}$ until required. We used sera for the assessment of blood urea nitrogen $(\mathrm{BUN})$ and serum creatinine $(\mathrm{sCr})$ to evaluate renal function [2]. These parameters were determined spectrophotometrically using commercial kits (Pars Azmoon, Tehran, Iran) as prescribed in the manufacturer's instructions provided with commercial kits.

2.7. Kidney Histological Examination. After the animals were euthanized, the right kidneys were isolated and washed with cold saline $(0.9 \% \mathrm{NaCl})$. After fixation in paraformaldehyde solution (10\%) for $24 \mathrm{~h}$ at RT, renal parts were dehydrated using ethanol. Paraffin-saturated tissues were preferred for further evaluation, and after $4 \mathrm{~h}$, the samples were fixed on microtome and cut in sections of $4 \mu \mathrm{m}$ thickness. Then, tissue slides were prepared and stained with hematoxylin-eosin (HE) [17]. Finally, two experienced morphologists randomly examined glomeruli; they were blinded to the origin of the slides.

\subsection{Evaluation of Oxidative Stress Parameters in Kidney Tissue}

2.8.1. Assessment of Glutathione Concentration in Kidney Tissue. An adequate glutathione (GSH) content is necessary in order to maintain the normal function of the kidneys. 
GSH is measured using DTNB (5, 5'-dithiobis-2-nitrobenzoic acid) as the indicator. Briefly, phosphate buffers and $1 \mathrm{ml}$ of the tissue homogenate were added to trichloroacetic acid. This mixture was centrifuged $1000 \times \mathrm{g}$ for 20 minutes; $1 \mathrm{ml}$ of supernatant was taken and added to $0.4 \%$ DTNB and $\mathrm{Na}_{2} \mathrm{PO}_{4}$ and incubated for 15 minutes to complete the reaction. We read the resulting yellow color at $412 \mathrm{~nm}$ using a UV spectrophotometer. Glutathione concentration was obtained as $\mu \mathrm{mol} / \mathrm{mL}$ (UV-1601 PC, Shimadzu, Japan) based on a calibration curve [18].

2.8.2. Measurement of Lipid Peroxidation Level in Kidney Tissue. Lipid peroxidation (LPO) was determined via quantifying malondialdehyde (MDA) concentrations, which were spectrophotometrically measured via the absorbance of the thiobarbituric acid (TBA) reaction product. In summary, phosphoric acid $(0.05 \mathrm{M})$ was added to tissue homogenate with the addition of $0.3 \mathrm{ml}$ thiobarbituric acid $(0.2 \%)$. When all tissue homogenates were prepared and previous steps were carried out, they were placed in a water bath $\left(100^{\circ} \mathrm{C}\right)$ for 30 minutes. Finally, the samples were taken to an ice bath, with the addition of $\mathrm{n}$-butanol to them. Thereafter, they were centrifuged at $3500 \times \mathrm{g}$ for 10 minutes. Finally, the MDA content was assessed by measuring the absorbance at $532 \mathrm{~nm}$ with Epoch $^{\mathrm{TM}}$ ELISA reader (Epoch ${ }^{\mathrm{TM}}$ Microplate Spectrophotometer, BioteK, USA) according to the calibration curve [19].

\subsection{Assessment of Mitochondrial Dysfunction}

2.9.1. Mitochondrial Preparation. Renal isolated mitochondria were obtained by differential centrifugation technique. The kidneys were homogenized with a glass homogenizer. Essentially, the homogenates were centrifuged for $10 \mathrm{~min}\left(\right.$ at $4^{\circ} \mathrm{C}, 2000 \times \mathrm{g}$ ). Then, the centrifugation of the supernatant was performed twice at $10,000 \times \mathrm{g}(10 \mathrm{~min})$. Residual mitochondrial pellets were washed and resuspended in cold tris buffer ( $\mathrm{pH} 7$ ). The mitochondrial preparation was performed on ice. Mitochondrial suspensions were used for the assessment of mitochondrial function and oxidative damage parameters [20].

2.9.2. Evaluation of Mitochondrial Function by Complex II Activity Assay. To investigate the mitochondrial function, the activity of succinate dehydrogenase (complex II) was assessed by the 3- [4, 5-dimethylthiazol-2-yl]-2, 5- diphenyltetrazoliumbromide (MTT) assay. Mitochondrial succinate dehydrogenase reduces MTT and produces formazan as a chromophoric product. After the isolation of mitochondria from the kidneys of mice, MTT (0.4\%) was added to the isolated mitochondria and incubated at $37^{\circ} \mathrm{C}$ (30 minutes). The produced formazan was dissolved in dimethyl sulfoxide (DMSO), and the absorbance was measured with Epoch $^{\mathrm{TM}}$ Microplate Spectrophotometer (BioteK, USA) at $570 \mathrm{~nm}$ [21].
2.9.3. Measurement of Glutathione Concentration in Isolated Renal Mitochondria. As previously explained, glutathione levels were evaluated by using DTNB as the indicator by spectrophotometry. Afterwards, $0.1 \mathrm{ml}$ of isolated renal mitochondria was added to phosphate buffers and DTNB $(0.04 \%)(\mathrm{pH} 7.4)$. The yellow color was read at $412 \mathrm{~nm}$ on a UV spectrophotometer (Lambda ${ }^{\text {TM }} 25$ UV-Vis, PerkinElmer, USA) [20].

2.9.4. Measurement of Lipid Peroxidation in Isolated Renal Mitochondria. Malondialdehyde (MDA) was measured based on the method used by Zamani et al. for the evaluation of lipid peroxidation [20]. Briefly, $0.25 \mathrm{ml}$ sulfuric acid $(0.05 \mathrm{M})$ was added to isolated renal mitochondria. In addition, $0.3 \mathrm{ml}$ TBA $(0.2 \%)$ was added. The tubes were kept in a water bath $\left(100^{\circ} \mathrm{C}\right)$ for 30 minutes. Lastly, the tubes were transferred to an ice bath, and $0.4 \mathrm{ml} \mathrm{n}$-butanol was added. Then, the samples were centrifuged for approximately 10 minutes at $3500 \times \mathrm{g}$. The supernatant absorbance was measured at $532 \mathrm{~nm}$ with Epoch ${ }^{\mathrm{TM}}$ ELISA reader Epoch $^{\mathrm{TM}}$ Microplate Spectrophotometer, BioteK, USA).

2.10. Measurement of Total Protein Concentration. Protein concentration was assessed in tissue homogenates by the Bradford technique. The samples were mixed with Coomassie blue; 10 minutes later, absorbance was determined at $595 \mathrm{~nm}$ (Lambda ${ }^{\mathrm{TM}} 25 \mathrm{UV}-\mathrm{Vis}$, PerkinElmer, USA) [22].

2.11. Statistical Analysis. All statistical analyses were performed using GraphPad Prism software, version 6. The results were expressed as mean \pm standard deviation. The assays were performed in at least triplicate, and the mean was used for statistical analysis. Comparison between groups was made using the one-way ANOVA test, followed by the post hoc Tukey's test. $P<0.05$ was considered statistically significant.

\section{Results}

3.1. Fasting Blood Glucose. Fasting blood glucose (FBG) is shown in Figure 1. As expected, the untreated diabetic mice had $292.7 \pm 19.66 \mathrm{mg} / \mathrm{dl}$, that is, about threefold higher than the control group $(P<0.001)$. Metformin dramatically decreased FBG compared with diabetic mice $(P<0.001)$. S. spinosa at $200 \mathrm{mg} / \mathrm{kg}$ significantly attenuated FBG compared with diabetic mice $(P<0.01)$; but it was not as effective as metformin-treated diabetic mice (Figure 1).

3.2. Kidney/Body Weight Coefficient. After 4-week treatment, we measured kidney weight/body weight in the groups. The untreated diabetic mice exhibited increased kidney/body weight $(P<0.001$ in comparison with control). On the other hand, treated diabetic mice with S. spinosa extract and metformin showed a significant weight loss compared to diabetic mice $(P<0.001)$. There were no 


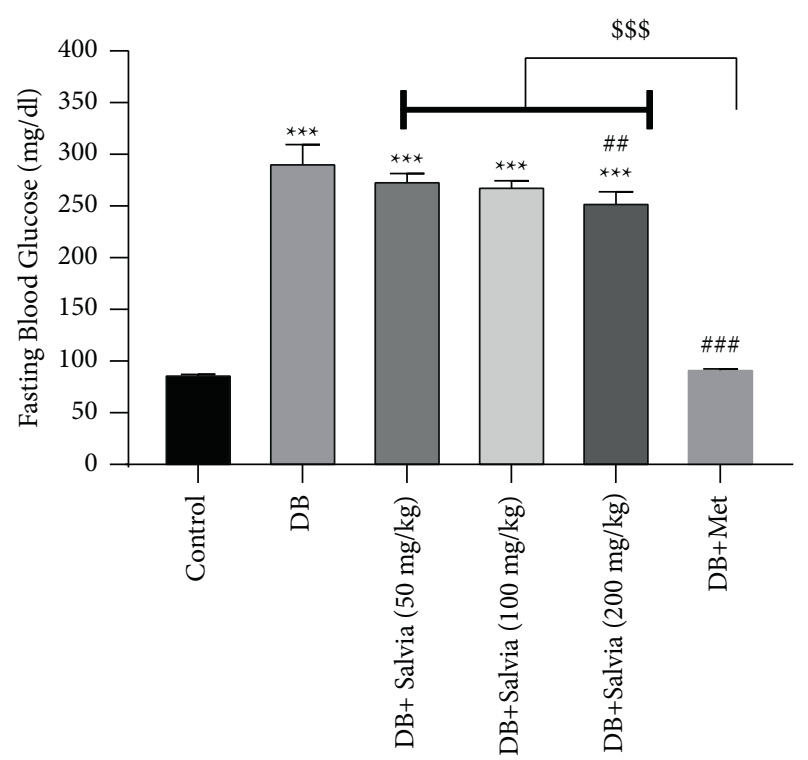

Figure 1: Effect of Salvia spinosa extracts on fasting blood glucose concentrations in streptozotocin-induced diabetic mice. Control: normal mice, DB: diabetic mice, DB + Salvia: diabetic mice given the methanolic extract of Salvia spinosa, and DB + Met: diabetic mice given metformin $(500 \mathrm{mg} / \mathrm{kg})$. Values represented as mean $\pm \mathrm{SD}$. ${ }^{* * *} P<0.001$ compared with control mice, ${ }^{\# \#} P<0.01$ compared with diabetic mice. ${ }^{\# \# \#} P<0.001$ compared with diabetic mice. $\$ \$ P<0.05$ compared with metformin-treated diabetic mice.

significant differences in metformin and S. spinosa (at 100 and $200 \mathrm{mg} / \mathrm{kg}$ )-treated diabetic mice groups (Figure 2).

3.3. Biochemical Parameters. As shown in Figure 3, the untreated diabetic mice showed increased BUN $(P<0.001)$ compared to control mice. S. spinosa and metformin decreased BUN concentrations remarkably compared to diabetic mice $(P<0.05)$. Interestingly, there are no significant changes in BUN concentrations in diabetic mice given S. spinosa (at $200 \mathrm{mg} / \mathrm{kg}$ ) compared to the metformin group $(P<0.01)$ (Figure 3).

Furthermore, serum creatinine ( $\mathrm{sCr}$ ), as a marker of GFR estimation, increased significantly in diabetic mice as compared to control mice $(P<0.01)$. The $S$. spinosa treatment considerably reduced $\mathrm{sCr}$ in diabetic mice so that the high doses of S. spinosa (100 and $200 \mathrm{mg} / \mathrm{kg}$ ) attenuated $\mathrm{sCr}$ in diabetic mice, almost as well as metformin-treated mice. Then, these data demonstrated that S. spinosa had a protective effect on kidney function in diabetic mice (Figure 4).

3.4. Effect of S. spinosa on Histopathology of the Kidney. Normal structures of glomeruli and renal tubular epithelial cells were clearly manifested in the kidney fractions of normal control mice (Figure 5(a)). The renal tissue of diabetic mice (Figure 5(b)) showed considerable glomerular and tubular damages represented by tubular necrosis with the vacuolar degeneration of proximal tubules, mononuclear cell infiltration, and thickened basement membranes. S. spinosa treatment for 4 weeks significantly ameliorated the severity of the degenerative changes in the renal tissue.

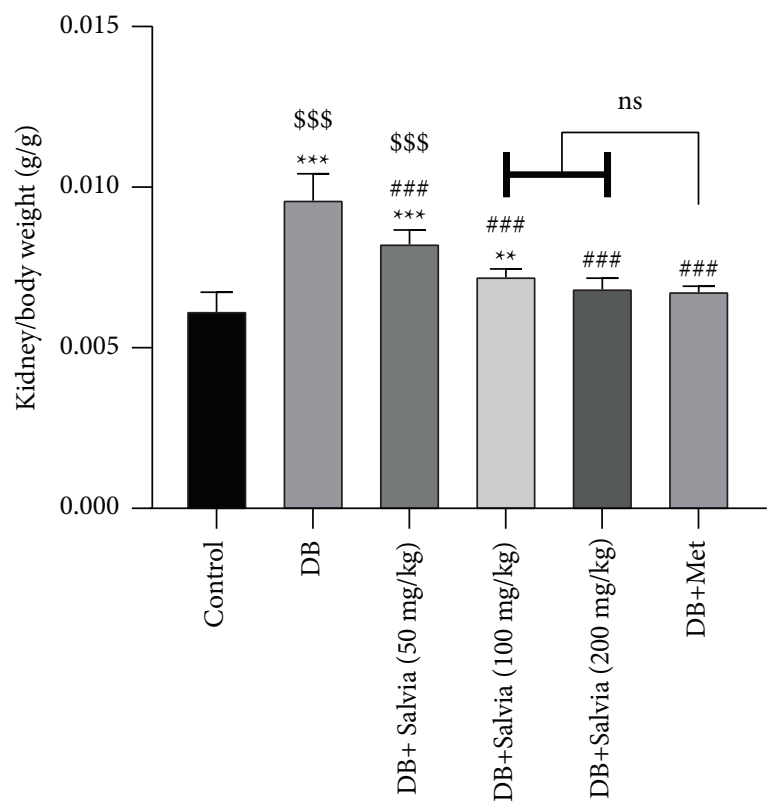

FIgURE 2: Effect of Salvia spinosa extracts on kidney/body weight coefficient in streptozotocin-induced diabetic mice. Control: normal mice, DB: diabetic mice, DB + Salvia: diabetic mice given the methanolic extract of Salvia spinosa, and DB + Met: diabetic mice given metformin $(500 \mathrm{mg} / \mathrm{kg})$. Values represented as mean \pm SD. ${ }^{* *} P<0.01$ compared with control mice, ${ }^{* * *} P<0.001$ compared with control mice, ${ }^{\# \# \# ~} P<0.001$ compared with diabetic mice. ${ }^{\$ \$} P<0.05$ compared with metformin-treated diabetic mice. Ns: nonsignificant.

Moreover, the mean glomerular volume of the normal control mice was significantly less than that of diabetic mice. The extract considerably reduced the mesangial expansion and glomerular volume compared to the diabetic mice (Figure 5(c)). Thus, our results indicated that S. spinosa extract alleviated mesangial expansion and glomerular hypertrophy.

\subsection{Oxidative Stress Damage in Kidney Tissue}

3.5.1. Glutathione Concentration in Kidney Tissue. Glutathione (GSH) is a main intrinsic antioxidant in tissues, and a decrease in its levels indicates oxidative damage. Figure 6 demonstrates that GSH concentrations in untreated diabetic mice were approximately half those of normal mice $(P<0.001)$. Treatment with $S$. spinosa at all concentrations increased this content significantly compared with diabetic mice $(P<0.001)$. Interestingly, there were no noteworthy differences between $S$. spinosa and metformin-treated mice (Figure 6).

3.5.2. Lipid Peroxidation in Kidney Tissue. Elevation of MDA (product of lipid peroxidation) is a main marker for oxidative stress. Similarly, in the current experience, diabetes led to an increase in MDA levels from $10.55 \pm 0.88 \mu \mathrm{M}$ in control group mice to $35.90 \pm 1.85 \mu \mathrm{M}$ in untreated diabetic mice (almost fourfold) $(P<0.001)$. Four-week treatment with $S$. spinosa significantly inhibited lipid peroxidation 


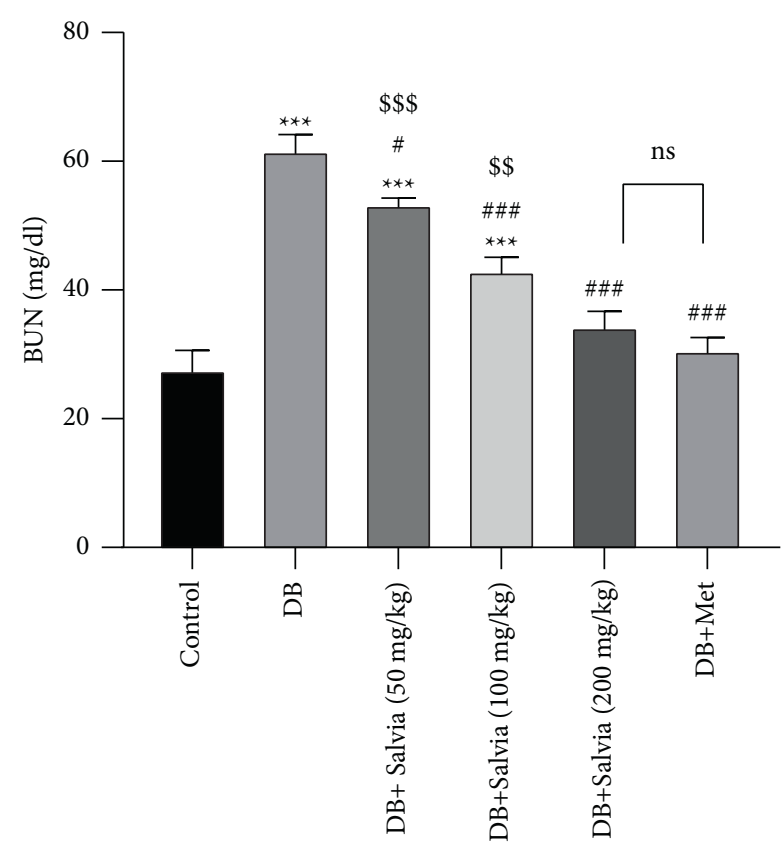

Figure 3: Effect of Salvia spinosa extracts on blood urea nitrogen (BUN) concentrations in streptozotocin-induced diabetic mice. Control: normal mice, DB: diabetic mice, DB + Salvia: diabetic mice given the methanolic extract of Salvia spinosa, and DB + Met: diabetic mice given metformin $(500 \mathrm{mg} / \mathrm{kg})$. Values represented as mean \pm SD. ${ }^{* * *} P<0.001$ compared with control mice. ${ }^{\#} P<0.05$ compared with diabetic mice. ${ }^{\# \#} P<0.01$ compared with diabetic mice. ${ }^{\# \# \# ~} P<0.001$ compared with diabetic mice. ${ }^{\$ \$} P<0.01$ compared with metformin-treated diabetic mice. ${ }^{\$ \$} P<0.05$ compared with metformin-treated diabetic mice. Ns: nonsignificant.

damage compared to the diabetic untreated group $(P<0.001)$. Intriguingly, significant changes were not seen between diabetic mice treated with S. spinosa $(200 \mathrm{mg} / \mathrm{kg})$ and metformin-treated mice (Figure 7).

\subsection{Mitochondrial Dysfunction}

3.6.1. Effect of S. spinosa on Mitochondrial Function (Complex II Activity). We also investigated S. spinosa effects on the activity of mitochondrial complex II (succinate dehydrogenase) via the MTT test. After the isolation of renal mitochondria, a significant reduction was observed in the mitochondrial metabolism of MTT to formazan in diabetic mice, compared with normal control mice $(P<0.001)$. $S$. spinosa clearly improved mitochondrial function in diabetic mice $(P<0.05)$. No significant difference was observed in S. spinosa-treated mice (100 and $200 \mathrm{mg} / \mathrm{kg}$ ) in comparison with metformin-treated mice (Figure 8).

3.6.2. Effect of S. spinosa on Glutathione Concentration of Isolated Renal Mitochondria. The GSH concentrations of diabetic mice were approximately $50 \%$ of control groups. As shown in Figure 9, the GSH levels were markedly elevated after S. spinosa treatment $(100$ and $200 \mathrm{mg} / \mathrm{kg})(P<0.05$ compared with diabetic mice). According to our results, $S$. spinosa represented a protective effect on mitochondrial

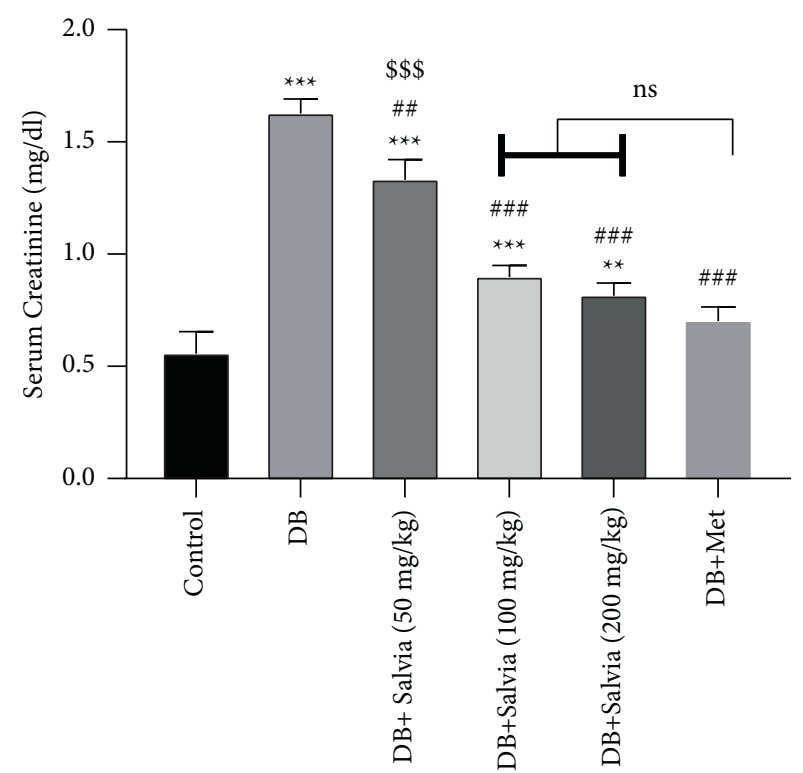

FIGURE 4: Effect of Salvia spinosa extracts on serum creatinine (sCr) concentrations in streptozotocin-induced diabetic mice. Control: normal mice, $\mathrm{DB}$ : diabetic mice, $\mathrm{DB}+$ Salvia: diabetic mice given the methanolic extract of Salvia spinosa, and DB + Met: diabetic mice given metformin $(500 \mathrm{mg} / \mathrm{kg})$. Values represented as mean $\pm \mathrm{SD}$. ${ }^{* *} P<0.01$ compared with control mice. ${ }^{* * *} P<0.001$ compared with control mice. \#\# $P<0.01$ compared with diabetic mice. ${ }^{\# \# \#} P<0.001$ compared with diabetic mice. ${ }^{\$ \$} P<0.05$ compared with metformin-treated diabetic mice. Ns: nonsignificant.

glutathione contents, specially at 100 and $200 \mathrm{mg} / \mathrm{kg}$ doses. Furthermore, the glutathione concentrations of metformintreated mice were significantly higher than those of diabetic mice $(P<0.05)$. (Figure 9$)$.

3.6.3. Effect of S. spinosa on Mitochondrial Lipid Peroxidation. As shown in Figure 10, MDA concentration was significantly increased in diabetic mice compared with normal mice $(P<0.05)$. Interestingly, $S$. spinosa-treated diabetic mice (at all doses) significantly decreased mitochondrial lipid peroxidation in diabetic mice $(P<0.05$ compared with untreated diabetic mice). The significant differences were not shown between S. spinosa- and metformin-treated groups (Figure 10).

\section{Discussion}

Diabetic nephropathy is considered the second most prevalent and severe complication of DM. Hypoglycemic, antihypertensive, and lipid-lowering drugs are the conventional medicines prescribed for $\mathrm{DM}$ and $\mathrm{DN}$, and these drugs are not totally satisfactory $[2,3,6,9]$. So, there is the necessity to look for new strategies and treatment for DN's complications. In this study, our focus was essentially on agents with better efficacy and minimum adverse effects.

Dietary and medicinal plants have shown incredible salutary effects on diabetic complications owing to their antioxidants. Herbal antioxidants can activate reduction- 


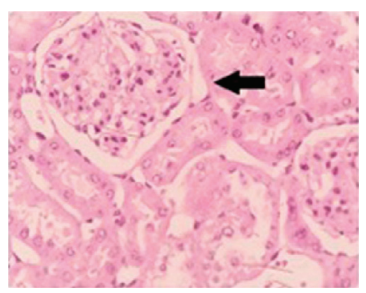

(a)

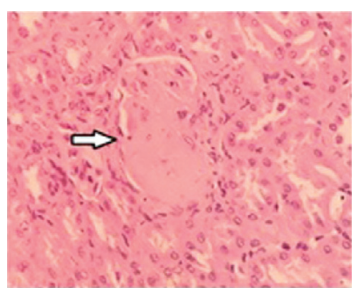

(b)

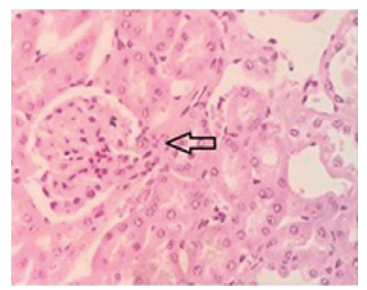

(c)

Figure 5: Hematoxylin- and eosin-stained sections of the kidney tissue $(\times 400)$. (a) Kidney from normal mice showing normal glomeruli (black arrow), (b) kidney from diabetic mice showing the structural disorganization in glomeruli (white arrow), and (c) kidney from S. spinosa $(200 \mathrm{mg} / \mathrm{kg})$-treated diabetic mice showing almost normal morphology.

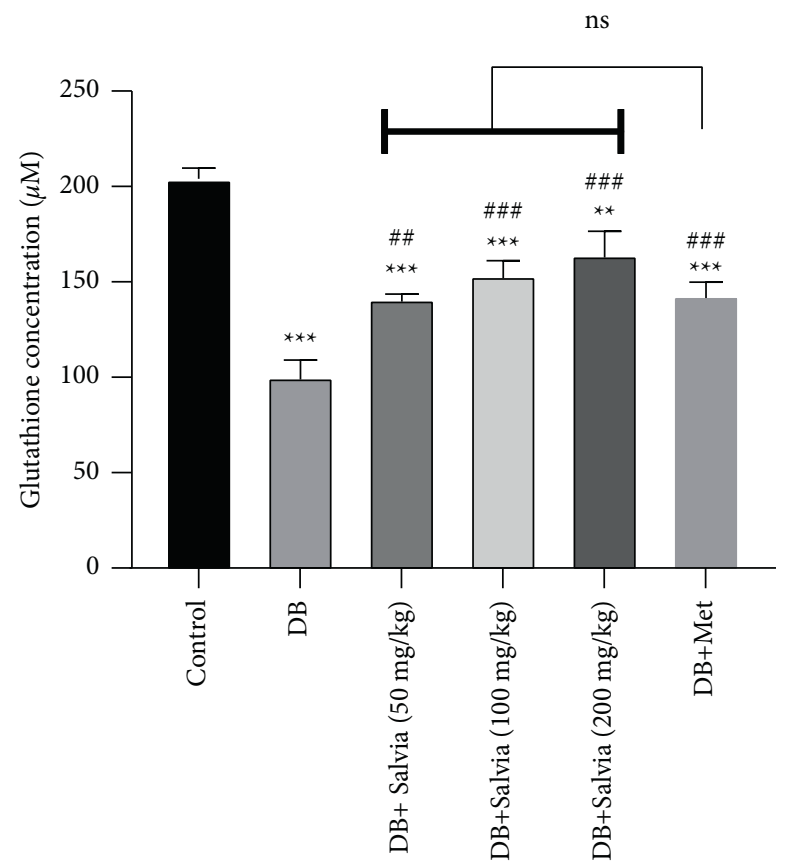

Figure 6: Effect of Salvia spinosa extracts on glutathione concentrations in the kidney tissue in streptozotocin-induced diabetic mice. Control: normal mice, DB: diabetic mice, DB + Salvia: diabetic mice given the methanolic extract of Salvia spinosa, and DB + Met: diabetic mice given metformin $(500 \mathrm{mg} / \mathrm{kg})$. Values represented as mean $\pm \mathrm{SD} .{ }^{* *} P<0.01$ compared with control mice. ${ }^{* * *} P<0.001$ compared with control mice. ${ }^{\# \# \# ~} P<0.001$ compared with diabetic mice. Ns: nonsignificant.

oxidation-sensitive transcription factors, and cellular antioxidant and cellular detoxification capabilities [10]. For example, the Croton hookeri extract dramatically reduced oxidative stress and renal histopathological damage due to its antioxidant capacity in diabetic rats [5]. As expected, in our study, the diabetic mice showed a rise in lipid peroxidation and GSH oxidation level in the kidney and isolated renal mitochondria, compared with the control group. Fourweek treatment with $S$. spinosa led to a significant improvement in DN. Similarly in recent years, Xiang et al. have found that $S$. miltiorrhiza has a therapeutic effect on several complications associated with DM, including DN due to antioxidant properties [3]. Numerous studies have shown that Salvia family plants contain numerous flavonoids such as 6, 8-di-C-glucosyl apigenin, apigenin 7-glucoside, luteolin 7-glucoside, luteolin 7-diglucoside, and phenolic acids. Available scientific data currently support beneficial effects of flavonoids and polyphenolic natural products on $\mathrm{DN}$
$[4,23]$. Flavonoids have been shown to possess phenomenal health-promoting effects. They demonstrated antioxidative and anti-inflammatory effects, and their capacity to modulate key cellular enzyme functions that are associated with antidiabetic properties $[4,10,24]$.

Renal hemodynamic changes, lipid disorders, polyol activation, oxidative stress, and inflammatory pathways are known to be several pathogeneses involved in diabetic nephropathy $[3,9,25]$. Moreover, one of the main causes of diabetic nephropathy is oxidative damage caused by persistent hyperglycemia, which can lead to renal mitochondrial dysfunction [10]. In our study, typical signs of DN, such as rise in serum creatinine, BUN, and glomerular hypertrophy, were observed in diabetic mice, and S. spinosa treatment provided renal protection by decreasing $\mathrm{sCr}$ and $\mathrm{BUN}$ as well as by providing antioxidant effects.

The renal cells have a high susceptibility to the hyperglycemia caused by diabetes. Although the complex 


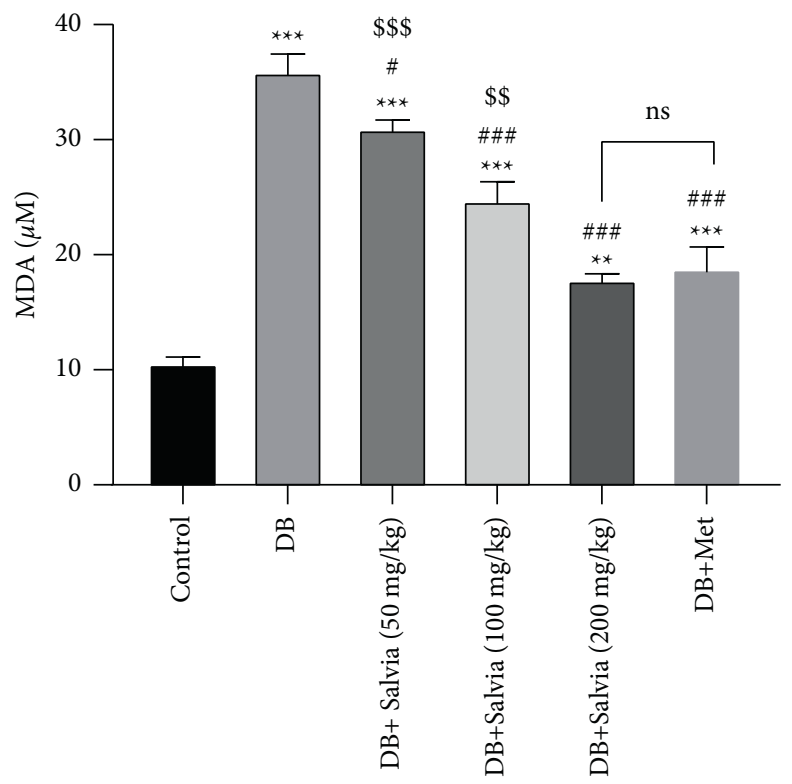

Figure 7: Effect of Salvia spinosa extracts on lipid peroxidation in the kidney tissue in streptozotocin-induced diabetic mice. Control: normal mice, DB: diabetic mice, DB + Salvia: diabetic mice given methanolic extract of Salvia spinosa, and DB + Met: diabetic mice given metformin $(500 \mathrm{mg} / \mathrm{kg})$. Values represented as mean $\pm \mathrm{SD}$. ${ }^{* * *} P<0.001$ compared with control mice. ${ }^{\#} P<0.05$ compared with diabetic mice. ${ }^{\# \#} P<0.01$ compared with diabetic mice. ${ }^{\# \# \#} P<0.001$ compared with diabetic mice. ${ }^{\$ \$} P<0.01$ compared with metformin-treated diabetic mice. ${ }^{\$ \$} P<0.05$ compared with metformin-treated diabetic mice. Ns: nonsignificant.

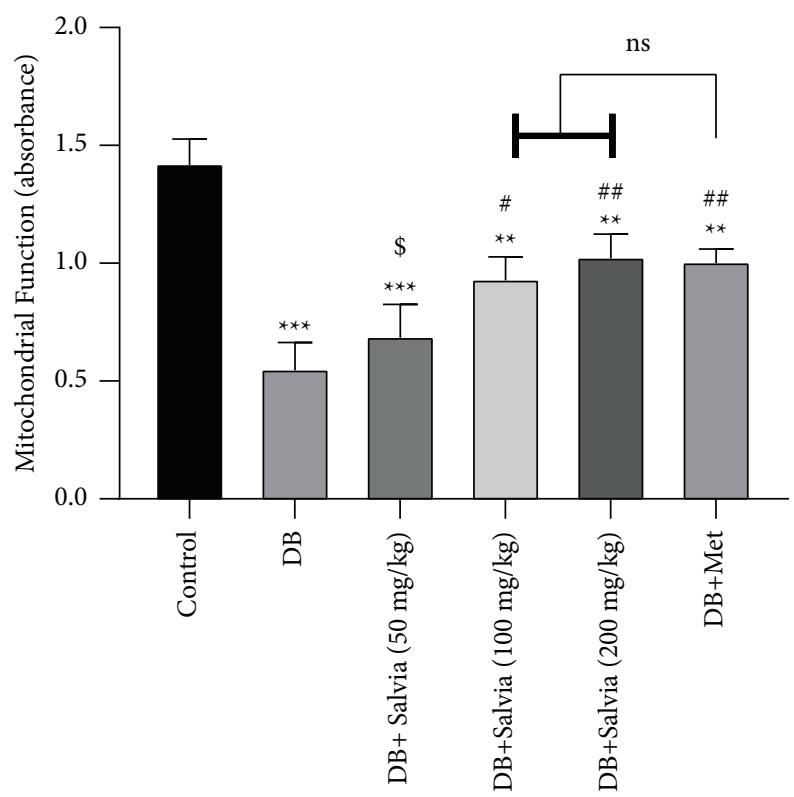

Figure 8: Effect of Salvia spinosa extracts on mitochondrial function in renal isolated mitochondria in streptozotocin-induced diabetic mice. Control: normal mice, DB: diabetic mice, DB + Salvia: diabetic mice given the methanolic extract of Salvia spinosa, and DB + Met: diabetic mice given metformin $(500 \mathrm{mg} / \mathrm{kg})$. Values represented as mean $\pm \mathrm{SD}$. ${ }^{* *} P<0.01$ compared with control mice. ${ }^{* * *} P<0.001$ compared with control mice. ${ }^{\#} P<0.05$ compared with diabetic mice. ${ }^{\# \#} P<0.01$ compared with diabetic mice. ${ }^{\$} P<0.05$ compared with metformin-treated diabetic mice. Ns: nonsignificant.

mechanisms underlying mitochondrial dysfunction in diabetic nephropathy are not fully assumed [1], hyperglycemia leads to an increase in intracellular glucose and acceleration of mitochondrial oxidative phosphorylation with the excessive leakage of single electrons to the oxygen molecule $\left(\mathrm{O}_{2}\right)$, thereby forming superoxide $\left(\mathrm{O}_{2}^{-}\right)$, leading to other reactive oxygen species (ROS). Minimal levels of ROS are detoxified by cellular antioxidants such as glutathione, catalase, and superoxide dismutase [26]. Hypothetically, excessive oxidative stress damages mitochondria, proteins, and membranes, resulting in mitoptosis (localized mitochondrial destruction). As demonstrated in our results, a 


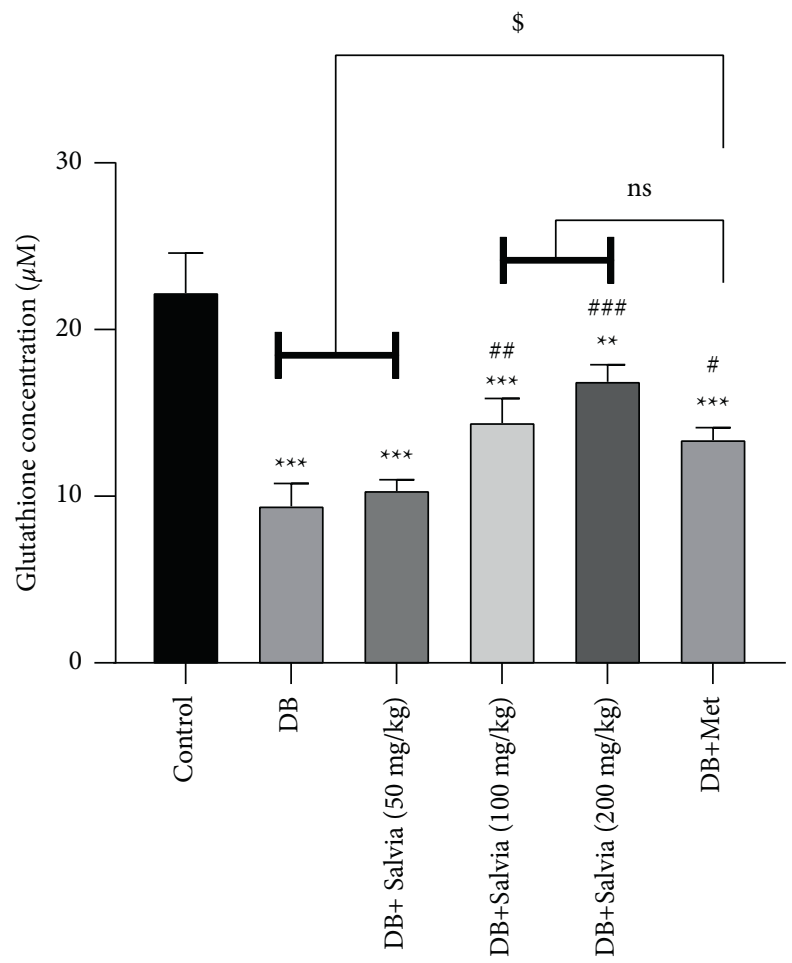

Figure 9: Effect of Salvia spinosa extracts on mitochondrial glutathione in renal isolated mitochondria in streptozotocin-induced diabetic mice. Control: normal mice, DB: diabetic mice, DB + Salvia: diabetic mice given the methanolic extract of Salvia spinosa, and DB + Met: diabetic mice given metformin $(500 \mathrm{mg} / \mathrm{kg})$. Values represented as mean $\pm \mathrm{SD}$. ${ }^{* *} P<0.01$ compared with control mice. ${ }^{* * *} P<0.001$ compared with control mice. ${ }^{\#} P<0.05$ compared with diabetic mice. ${ }^{\# \#} P<0.01$ compared with diabetic mice. ${ }^{\# \#} P<0.001$ compared with diabetic mice. ${ }^{\$} P<0.05$ compared with metformin-treated diabetic mice. Ns: nonsignificant.

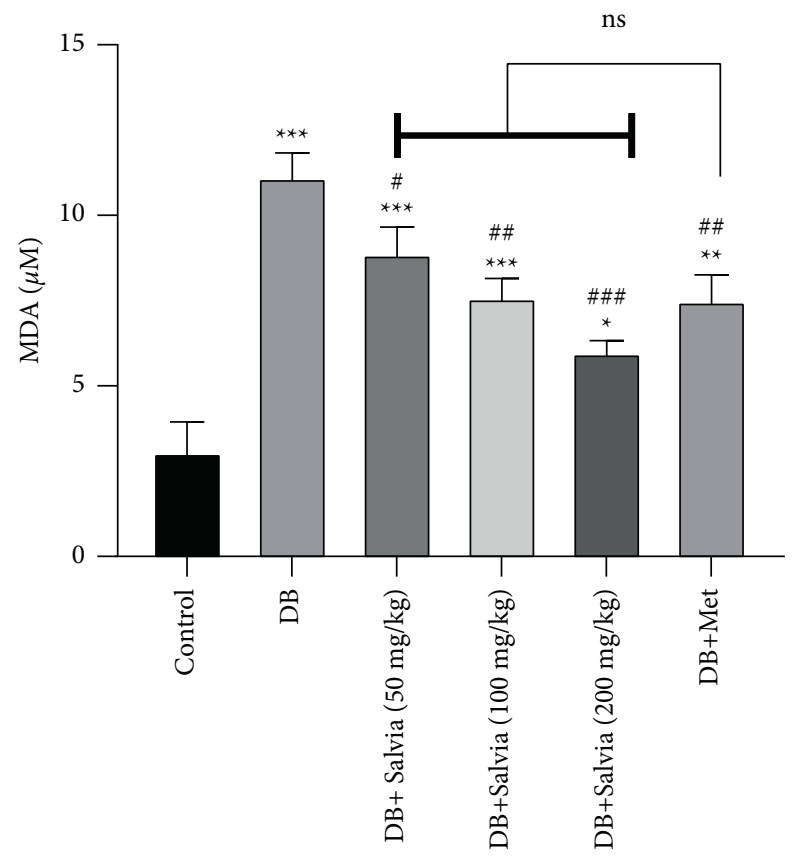

FIGURE 10: Effect of Salvia spinosa extracts on mitochondrial lipid peroxidation in renal isolated mitochondria in streptozotocin-induced diabetic mice. Control: normal mice, DB: diabetic mice, DB + Salvia: diabetic mice given the methanolic extract of Salvia spinosa, and $\mathrm{DB}+$ Met: diabetic mice given metformin $(500 \mathrm{mg} / \mathrm{kg})$. Values represented as mean $\pm \mathrm{SD} .{ }^{*} P<0.05$ compared with control mice. ${ }^{* *} P<0.001$ compared with control mice. ${ }^{* * *} P<0.001$ compared with control mice. ${ }^{\#} P<0.05$ compared with diabetic mice. ${ }^{\# \#} P<0.01$ compared with diabetic mice. ${ }^{\# \# \#} P<0.001$ compared with diabetic mice. Ns: nonsignificant. 
significant plummet in mitochondrial function was observed in diabetic mice compared to the control group, and we have indicated that $S$. spinosa led to a remarkable amelioration of mitochondrial toxicity [10]. In addition, lipid peroxidation decreased, and GSH amounts normalized (in kidneys and isolated renal mitochondria) in 4 weeks of treatment. Similarly, other studies demonstrated that the antioxidants such as coenzyme Q10 had a nephroprotective effect in DN by preventing mitochondrial oxidative damage. Therefore, as shown in results, $S$. spinosa reduced the level of oxidative stress and led to mitochondrial protecting effects in the presence of diabetes-induced mitochondrial damage.

In addition, the ROS along with oxidized proteins, lipids, nucleic acids, and carbohydrates contribute to cellular dysfunction and compromised integrity, and eventually to apoptosis (programmed cell death) [26]. These lead to such histopathological changes as glomerular hypertrophy and promote fibrogenesis in the glomeruli and tubules [25]. Furthermore, our results of kidney histopathology revealed the alleviated glomerular hypertrophy and mesangial expansion in diabetic mice, showing that these damages were improved after $S$. spinosa treatment. Similar to our results, Trujillo et al. showed that antioxidant plants such as turmeric had nephron-protective effects due to the inhibition of mitochondrial dysfunction and prevention of oxidative stress [27], as well as preventing lipid peroxidation [28, 29]. Thus, S. spinosa can probably alleviate diabetic nephrotoxicity via protecting mitochondria, by reducing oxidative stress. S. spinosa combined with antihyperglycemic agents could be highly effective in the treatment of diabetic nephropathy. Nevertheless, the lack of phytochemical analysis is a limitation of our study. As previous data suggest, the quality of plant materials is associated with the geographic and ecological conditions and phytochemical analysis should be carried out in subsequent studies.

\section{Conclusions}

In summary, advanced oxidative stress is a probable mechanism, which contributes to the diabetic complications, including nephropathy. To the best of our knowledge, our study has shown for the first time that Salvia spinosa effectively decreased oxidative stress and renal injury in STZ-induced diabetic mice. S. spinosa reduced the levels of sCr and BUN and improved the histopathological state in the diabetic mice. Moreover, S. spinosa significantly prevented mitochondrial dysfunction. The results suggest that in the treatment of diabetic nephropathy, S. spinosa is a potential therapeutic agent. Therefore, it is necessary to carry out further exploration for authentic data to justify clinical application of this plant against diabetic complications, besides other blood glucose-lowering protocols.

\section{Data Availability}

Data of this study are available from the corresponding author upon reasonable request.

\section{Disclosure}

The data provided in this research were extracted from a part of the Pharm D thesis.

\section{Conflicts of Interest}

The authors declare no conflicts of interest.

\section{Acknowledgments}

This research was supported by the Research Council of Guilan University of Medical Sciences, Rasht, Iran (Registration CODE: IR.GUMS.REC.1398.358).

\section{References}

[1] C. C. Khoury, S. Chen, and F. N. Ziyadeh, "Pathophysiology of diabetic nephropathy," Chronic Renal Disease, pp. 279-296, 2020.

[2] C. Guo, Y. Dong, H. Zhu, Y. Liu, and G. Xie, "Ameliorative effects of protodioscin on experimental diabetic nephropathy," Phytomedicine, vol. 51, pp. 77-83, 2018.

[3] X. Xiang, H.-D. Cai, S.-L. Su et al., "Salvia miltiorrhiza protects against diabetic nephropathy through metabolome regulation and wnt $\beta$-catenin and TGF- $\beta$ signaling inhibition," Pharmacological Research, vol. 139, pp. 26-40, 2019.

[4] T. Caro-Ordieres, G. Marín-Royo, L. Opazo-Ríos et al., “The coming age of flavonoids in the treatment of diabetic complications," Journal of Clinical Medicine, vol. 9, no. 2, p. 346, 2020.

[5] A. Kundu, P. Dey, P. Sarkar et al., "Protective effects of Croton hookeri on streptozotocin-induced diabetic nephropathy," Food and Chemical Toxicology, vol. 135, Article ID 110873, 2020.

[6] S. Sifuentes-Franco, D. E. Padilla-Tejeda, S. Carrillo-Ibarra, and A. G. Miranda-Díaz, "Oxidative stress, apoptosis, and mitochondrial function in diabetic nephropathy," The Internet Journal of Endocrinology, vol. 2018, Article ID 1875870, 13 pages, 2018

[7] M. Shokrzadeh, S. Sadat-Hosseini, M. Fallah, and F. Shaki, "Synergism effects of pioglitazone and Urtica dioica extract in streptozotocin-induced nephropathy via attenuation of oxidative stress," Iranian Journal of Basic Medical Sciences, vol. 20, no. 5, pp. 497-502, 2017.

[8] M. B. Bahadori, H. Valizadeh, B. Asghari, L. Dinparast, M. Moridi Farimani, and S. Bahadori, "Chemical composition and antimicrobial, cytotoxicity, antioxidant and enzyme inhibitory activities of Salvia spinosa L," Journal of Functional Foods, vol. 18, pp. 727-736, 2015.

[9] L. Tang, K. Li, Y. Zhang et al., "Quercetin liposomes ameliorate streptozotocin-induced diabetic nephropathy in diabetic rats," Scientific Reports, vol. 10, no. 1, pp. 2440-2448, 2020.

[10] N. Al-Waili, H. Al-Waili, T. Al-Waili, and K. Salom, "Natural antioxidants in the treatment and prevention of diabetic nephropathy; a potential approach that warrants clinical trials," Redox Report, vol. 22, no. 3, pp. 99-118, 2017.

[11] M. B. Bahadori, L. Dinparast, G. Zengin et al., "Functional components, antidiabetic, anti-Alzheimer's disease, and antioxidant activities of Salvia syriaca L.," International Journal of Food Properties, vol. 20, no. 8, pp. 1761-1772, 2017.

[12] K. Miura, H. Kikuzaki, and N. Nakatani, "Antioxidant activity of chemical components from sage (Salvia officinalis L.) and 
thyme (Thymus vulgaris L.) measured by the oil stability index method," Journal of Agricultural and Food Chemistry, vol. 50, no. 7, pp. 1845-1851, 2002.

[13] M. Huang, Y. Xie, L. Chen et al., "Antidiabetic effect of the total polyphenolic acids fraction from Salvia miltiorrhiza bunge in diabetic rats," Phytotherapy Research, vol. 26, no. 6, pp. 944-948, 2012.

[14] A. Dweck, "The folklore and cosmetic use of various Salvia species," Sage The Genus Salvia, vol. 14, pp. 1-25, 2000.

[15] I. E. M. Zabad, M. N. Amin, and M. M. El-Shishtawy, "Protective effect of vanillin on diabetic nephropathy by decreasing advanced glycation end products in rats," Life Sciences, vol. 239, Article ID 117088, 2019.

[16] J. E. Ayala, V. T. Samuel, G. J. Morton et al., "Standard operating procedures for describing and performing metabolic tests of glucose homeostasis in mice," Disease Models \& Mechanisms, vol. 3, no. 9-10, pp. 525-534, 2010.

[17] C. Guo, C. Li, Y. Yu, W. Chen, T. Ma, and Z. Zhou, "Antihyperglycemic and antihyperlipidemic activities of protodioscin in a high-fat diet and streptozotocin-induced diabetic rats," RSC Advances, vol. 6, no. 91, pp. 88640-88646, 2016.

[18] A. Azari, M. Shokrzadeh, E. Zamani, N. Amani, and F. Shaki, "Cerium oxide nanoparticles protects against acrylamide induced toxicity in HepG2 cells through modulation of oxidative stress," Drug and Chemical Toxicology, vol. 42, no. 1, pp. 54-59, 2019.

[19] F. Shaki, M. Arab-Nozari, P. Elahi, M. Ghasemi, and E. Habibi, "Ameliorative effect of Viola odorata L. ethyl acetate extract against nephrotoxicity induced by chronic ethanol exposure in rats," Journal of Mazandaran University of Medical Sciences, vol. 29, no. 175, pp. 1-13, 2019.

[20] E. Zamani, F. Shaki, S. AbedianKenari, and M. Shokrzadeh, "Acrylamide induces immunotoxicity through reactive oxygen species production and caspase-dependent apoptosis in mice splenocytes via the mitochondria-dependent signaling pathways," Biomedicine \& Pharmacotherapy, vol. 94, pp. 523-530, 2017.

[21] M. Shokrzadeh, E. Zamani, J. Mollahasani, and F. Shaki, "Captopril inhibited metamphetamine-induced cardiac mitochondrial damage in hyperthermic condition via modulation of biochemical markers," Koomesh, vol. 18, no. 1, 2016.

[22] E. Zamani, M. Mohammadbagheri, M. Fallah, and F. Shaki, "Atorvastatin attenuates ethanol-induced hepatotoxicity via antioxidant and anti-inflammatory mechanisms," Research in Pharmaceutical Sciences, vol. 12, no. 4, pp. 315-321, 2017.

[23] M. E. Abreu, M. Müller, L. Alegre, and S. Munné-Bosch, "Phenolic diterpene and $\alpha$-tocopherol contents in leaf extracts of 60 Salvia species," Journal of the Science of Food and Agriculture, vol. 88, no. 15, pp. 2648-2653, 2008.

[24] L. Zhao, L. Xu, X. Tao et al., "Protective effect of the total flavonoids from Rosa laevigata michx fruit on renal ischemiareperfusion injury through suppression of oxidative stress and inflammation," Molecules, vol. 21, no. 7, p. 952, 2016.

[25] C. C. Khoury, S. C. Khourya, S. Chen, and F. N. Ziyadeh, "Pathophysiology of diabetic nephropathy," in Chronic Renal Disease, pp. 151-162, Elsevier, Amsterdam, Netherlands, 2015.

[26] J. L. Edwards, A. M. Vincent, H. T. Cheng, and E. L. Feldman, "Diabetic neuropathy: mechanisms to management," Pharmacology \& Therapeutics, vol. 120, no. 1, pp. 1-34, 2008.

[27] J. Trujillo, Y. I. Chirino, E. Molina-Jijón, A. C. AndéricaRomero, E. Tapia, and J. Pedraza-Chaverrí, "Renoprotective effect of the antioxidant curcumin: recent findings," Redox Biology, vol. 1, no. 1, pp. 448-456, 2013.

[28] S. Reddy and B. B. Aggarwal, "Curcumin is a non-competitive and selective inhibitor of phosphorylase kinase," FEBS Letters, vol. 341, no. 1, pp. 19-22, 1994.

[29] W. Wu, H. Geng, Z. Liu, H. Li, and Z. Zhu, "Effect of curcumin on rats/mice with diabetic nephropathy: a systematic review and meta-analysis of randomized controlled trials," Journal of Traditional Chinese Medicine, vol. 34, no. 4, pp. 419-429, 2014. 